\title{
Shuffle Reduction Based Sparse Matrix-Vector Multiplication on Kepler GPU
}

\author{
Yuan Tao ${ }^{1}$ and Huang Zhi-Bin ${ }^{2}$ \\ ${ }^{1}$ College of Mathematics, Jilin Normal University, Siping Jilin 136000, China \\ ${ }^{2}$ Beijing Key Lab of Intelligent Telecommunication Software and Multimedia,Beijing \\ University of Posts and Telecommunications, Beijing China,100876 \\ 'taoyuanjl@foxmail.com \\ ${ }^{2}$ Huangzb@bupt.edu.cn
}

\begin{abstract}
GPU is the suitable equipment for accelerating computing-intensive applications in order to get the higher throughput for High Performance Computing (HPC). Sparse Matrix-Vector Multiplication (SpMV) is the core algorithm of HPC, so the SpMV's throughput on GPU may affect the throughput on HPC platform. In the paper, we focus on the latency of reduction routine in SpMV included in CUSP, such as accessing shared memory and bank conflicting while multiple threads simultaneously accessing the same bank. We provide shuffle method to reduce the partial results instead of reducing in the shared memory in order to improve the throughput of SpMV on Kepler GPU. Experiments show that shuffle method can improve the throughput up to $9 \%$ of the original routine of SpMV in CUSP on average.
\end{abstract}

Keywords: gpu; sparse matrix; vector; shuffle; reduction

\section{Introduction}

Sparse matrix can reflect the exact properties of some applications and is widely used in HPC [1]. SpMV is the core algorithm of most sparse matrix computing, the throughput of SpMV on GPU may impact the performance of HPC immediately. GPU has been widely used in HPC to accelerate the computing-intensive algorithms, because it could provide much more throughput than CPU for some applications, many researchers and scientists have done a lot of research for SpMV on GPU and got some prefer results.

The seminal work of Nathan Bell [2] could get 16 GFLOPS on single GPU. The primary contribution is that different threads store the partial sum of productions in the shared memory and reduce in the shared memory for the final result by means of the different warp switching and this can avoid explicit synchronized operation, so we can get much more throughput on GPU. Limited to the GPU's architecture, there may be the much more latency while each thread accessing the shared memory or meeting bank conflict during one more threads concurrently accessing the same bank of the shared memory at the same time, the two factors above should not be ignored for current GPU for higher throughput. In the paper we focus on the issues on Kepler GPU, experiments show that the proposed method can provide at most $9 \%$ much more throughput than the original one of CUSP.

The paper is organized as followed. Section 2 gives the preliminaries of this work. In section 3 we provide shuffle reduction based CSR's SpMV on GPU. Section 4 reports the experimental result and discussion. At last we get conclusion in section 5. 


\section{Preliminaries}

\subsection{General Purpose Computing with GPU}

The original GPU is used for rendering the graphics. Being pursuit for higher computing throughput of HPC, programmability has been made by hardware vendors, such as NVIDIA and AMD. In the paper, we used NVIDIA GPU and programming model, CUDA, for our experiments.

There are three ties for the CUDA's program. A thread is a basic parallel unit, multiple threads constitute a block, and multiple blocks constitute a grid, however multiple grids should be launched one more times. A CUDA program is Single Program Multiple Data (SPMD), warp of CUDA is the basic unit to be launched, a warp is consisted of 32 threads, the thread ID within warp is called lane, one block may be consisted of multiple warps. Different warp in the same block should be required to be employed in the same Stream Multi-processor (SM), and different blocks may be employed in the same SM too. Upon the current CUDA's data organization, the threads in the same block can share the data with shared memory, however the explicit synchronized command should be required in CUDA for most applications. Bank conflict will meet while multiple threads accessing the same bank at the same time, this will introduce additional latency overhead. All the threads should exchange data by means of global memory. For Kepler GPU, the threads in the same warp can exchange register's value directly by means of shuffle command [3], which can save to access the shared memory.

\subsection{Compressed Sparse Row}

Figure 1, shows the format of Compressed Sparse Row (CSR) for sparse matrix. It means to store the non-zeros along the row, such as val for storing the non-zeros, the length of col_ptr is the same as val, and the col_ptr is to store the non-zero's column, row_ptr is the position of first non-zeros of each row, its length is rows+1, in which rows is the row's number of sparse matrix. The method can save to store the row index of each non-zero, so it can save much more storage and communication overhead.

\begin{tabular}{l}
\hline $\mathrm{T} *$ val $\quad$ //Non-zeros of sparse matrix \\
integer * col_ptr //Column's index of non-zeros \\
integer * row_ptr //Row pointer
\end{tabular}

Figure 1. CSR Structure

\subsection{Shared Memory Reducing Based SpMV}

GPU is a parallel platform, different logic cores compute different elements in parallel (or we call threads in CUDA). For SpMV, the general routine is that all the threads within a warp should iterate along a non-zeros row, we should do reduction after the threads finished the iterating all the non-zeros of current row.

Figure 2, lists pseudo-code of CSR based SpMV on GPU which is provided by CUSP [2], the library of CUSPARSE [4] is the extended of CUSP. It launches the fixed number of threads, such as the max number of threads, and one warp compute a row of sparse matrix, the threads in the warp iterate all the non-zeros in a row (line 12 and 13). After the warp finished the current row, the threads store the partial sum of products into the shared memory (line 14), and they do the reduction for the shared memory from line 15 to line 24 . They store the final result once they finished computing (line 25 and line 26). After all the warps iterate the rows of sparse matrix (line 8), the program is over. 


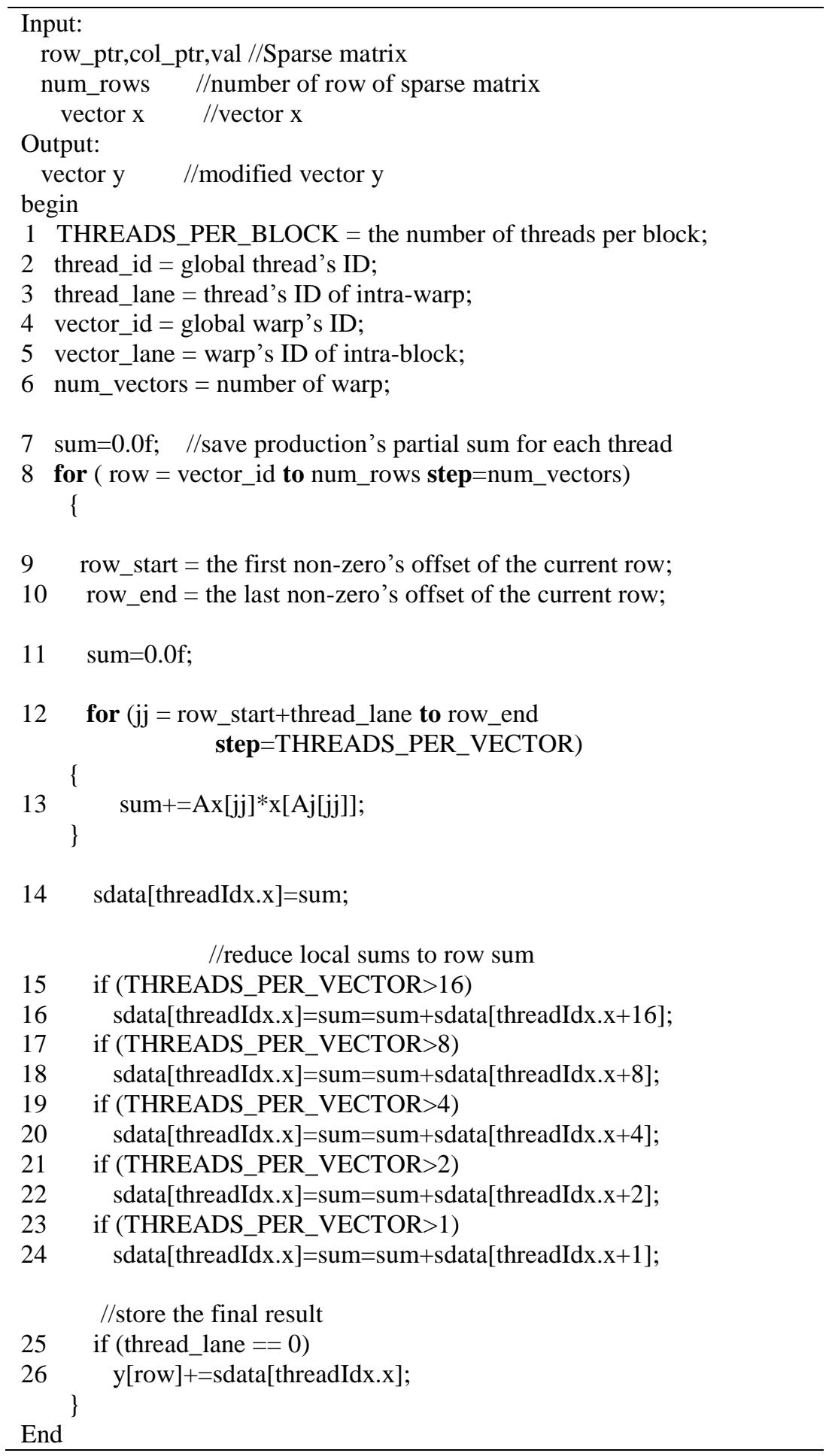

Figure 2. Pseudo-Code of Shared Memory Based Reduction for SpMV on GPU

\section{Shuffle Reduction Based CSR's SpMV on GPU}

Reduction is required for SpMV in parallel because the partial sum is stored in distribution, the key is how to access and sum them in a short time. For Kepler, we can use the shuffle command to exchange the register's value within the warp, this can avoid of accessing the shared memory, and save the bank conflicts which will bring much more latency. We will discuss the reduction for SpMV in parallel with shuffle in the section. 
As Figure 3, shows that we use the __shfl_xor() to reduce the different threads' partial sum [3], which are from line 14 to line 23, such as line 15-f is single-precision data and $15-\mathrm{d}$ is double-precision data. However double-precision data is not supported by __shfl_xor(), we resort to PTX instruction [5], which is named __shfl().

The double-precision data's implementation's details are provided in Figure 4. We convert double-precision data into two integers at line 3, which are high bits and low bits, and do _shfl_xor() for them respectively which are at line 4 and line 5, at last we convert high bits and low bits into a double-precision data at line 6 , and then return the result.

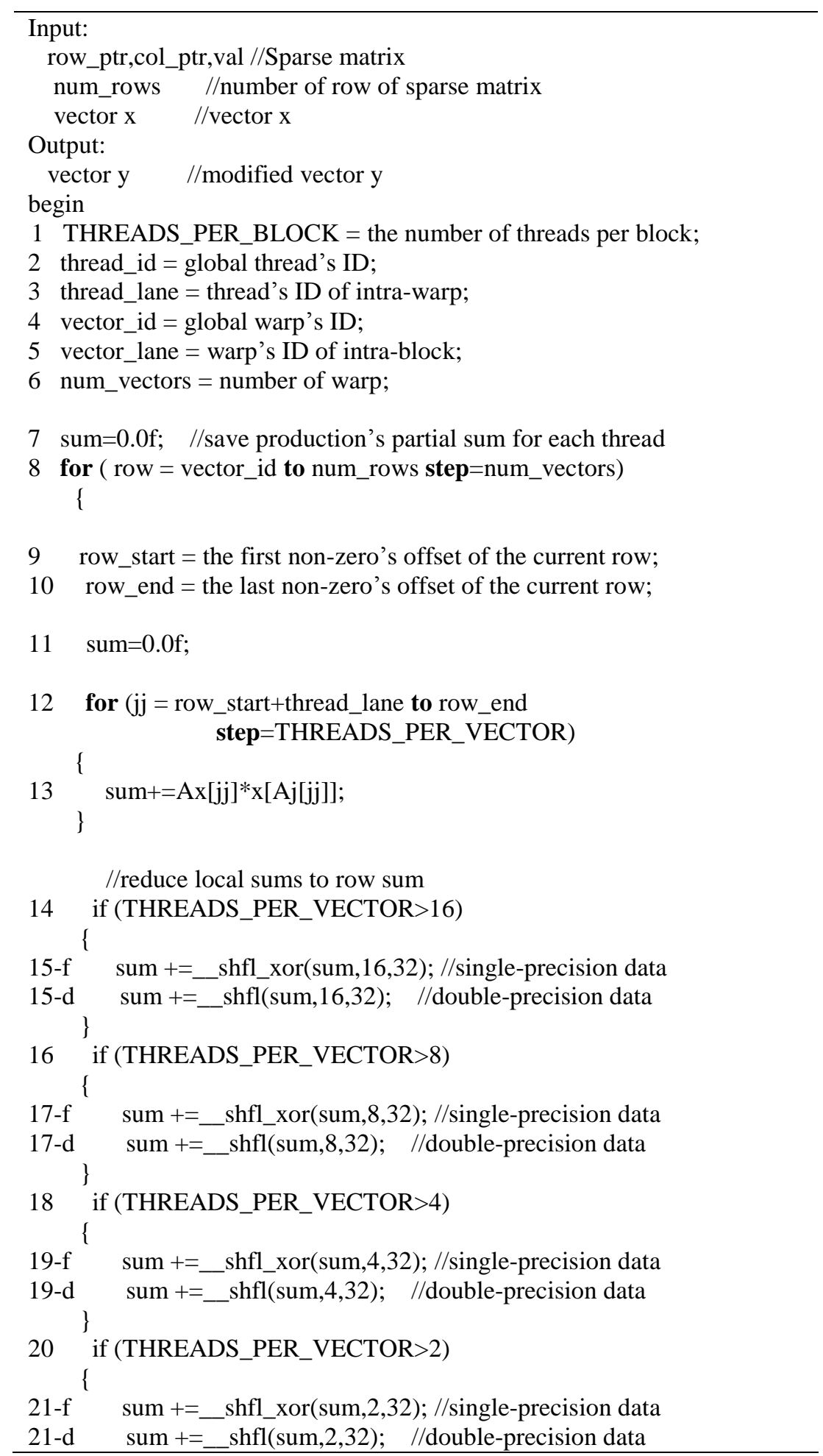




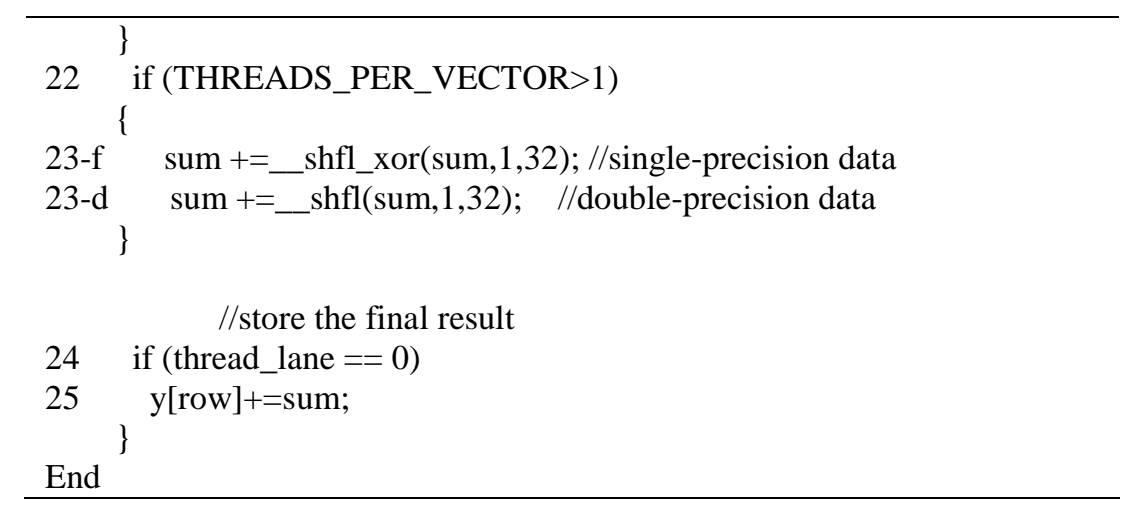

Figure 3. Shuffle Based Reduction for SpMV on GPU

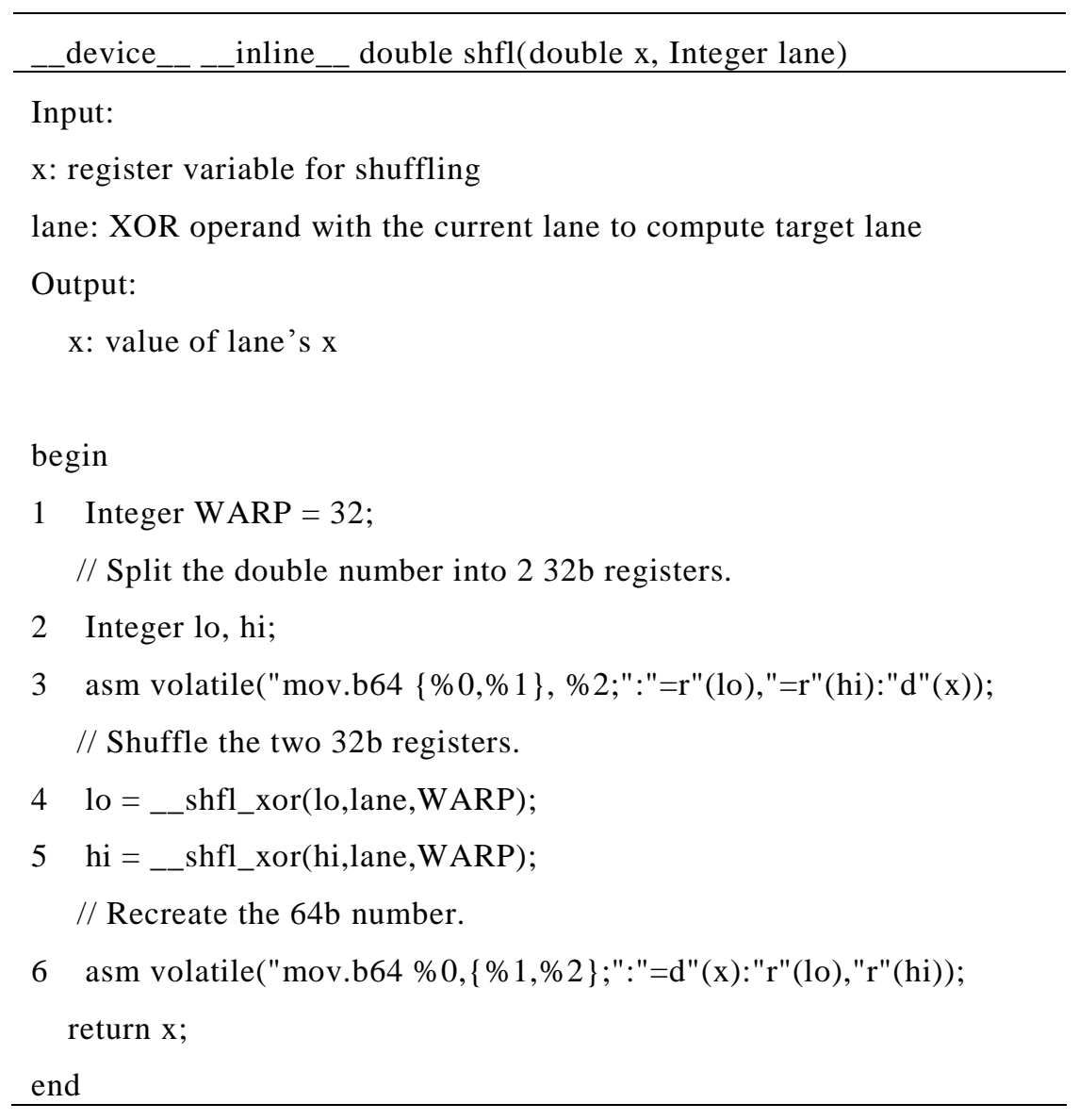

Figure 4. Shuffle for Double Precision Data on GPU [5]

\section{Experimental Results and Discussion}

In the section, we evaluate the effectiveness of our shuffle reduction, and compare with the SpMV routine of CUSP.

\subsection{Experimental Setup}

In this work, all the experiments are done on Kepler GPU listed in Table 1. All the benchmarking matrices are downloaded from Florida University [6], and most matrices are used by Nathan Bell et. al., [2], in the related work, which are provided in Table 2. 
Table 1. Test platform used in this work

\begin{tabular}{ccc}
\hline Platform & GPU & CPU \\
\hline Name & K20M & Intel(R) Xeon(R) CPU E5-2620 \\
Architecture & Kepler & x86_64 \\
Compute & 3.5 & N/A \\
Capability & $13 \times 192=2496$ & 4 way 6 cores \\
Cores & CUDA 5.0 & gcc 4.4.6 \\
Compiler and & Runtime & Red Hat Enterprise Linux Server release 6.2 X86_64 \\
Operation System & & 2.6.32-220.el6.x86_64 \\
Kernel
\end{tabular}

Table 2. Benchmarking Matrices Used in this Work

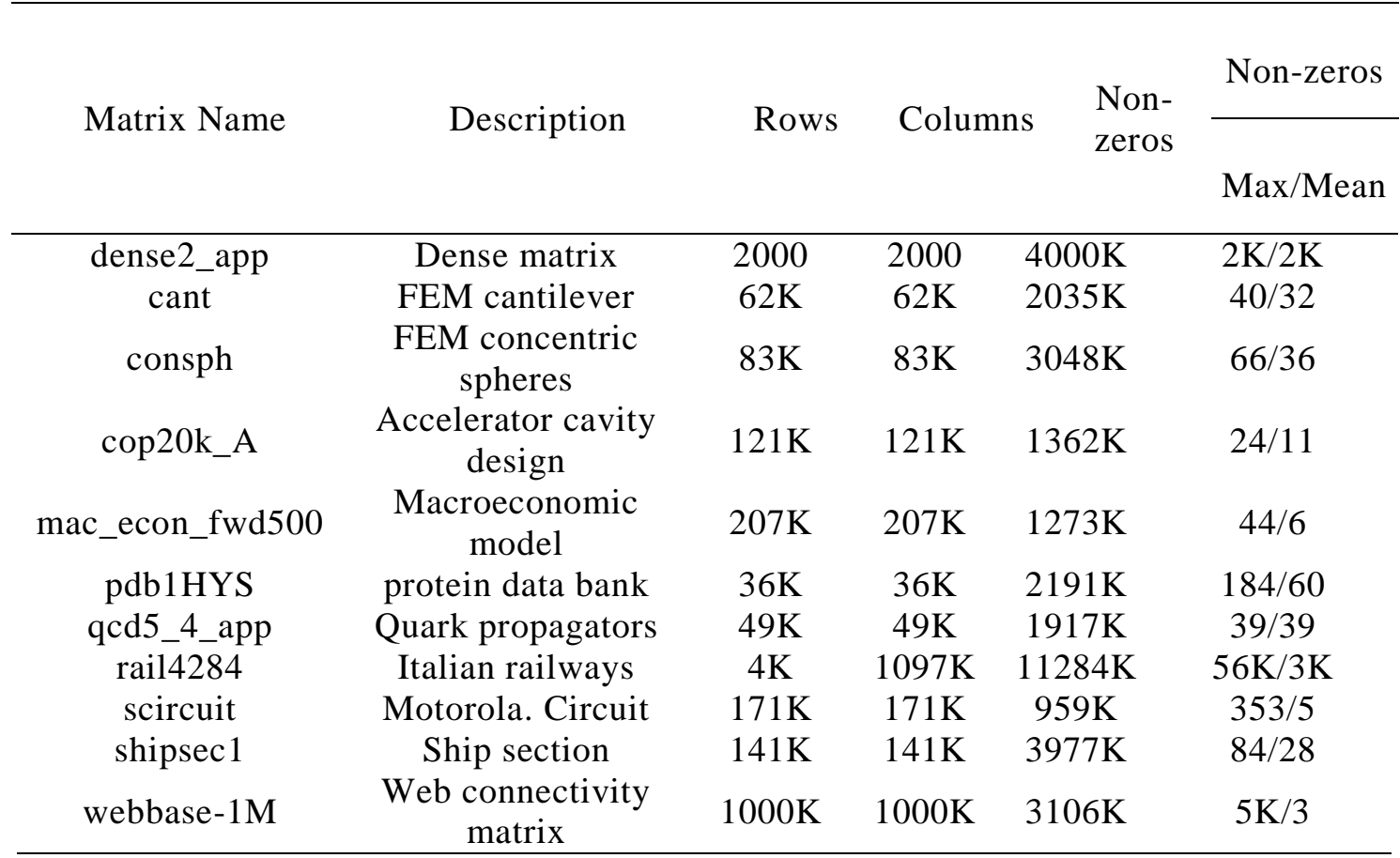

\subsection{Experimental Results and Discussion}

Figure 3, and Figure 4, give the single precision data and double precision data throughput on GPU respectively. From the experimental result we can get that our shuffle reduction implementation are surpass the reduction with shared memory for all benchmarking matrices. On the average, our shuffle reducing surpass the reduction with shared memory for single precision data by $5.3 \%$ and for double precision data by $9.1 \%$. 


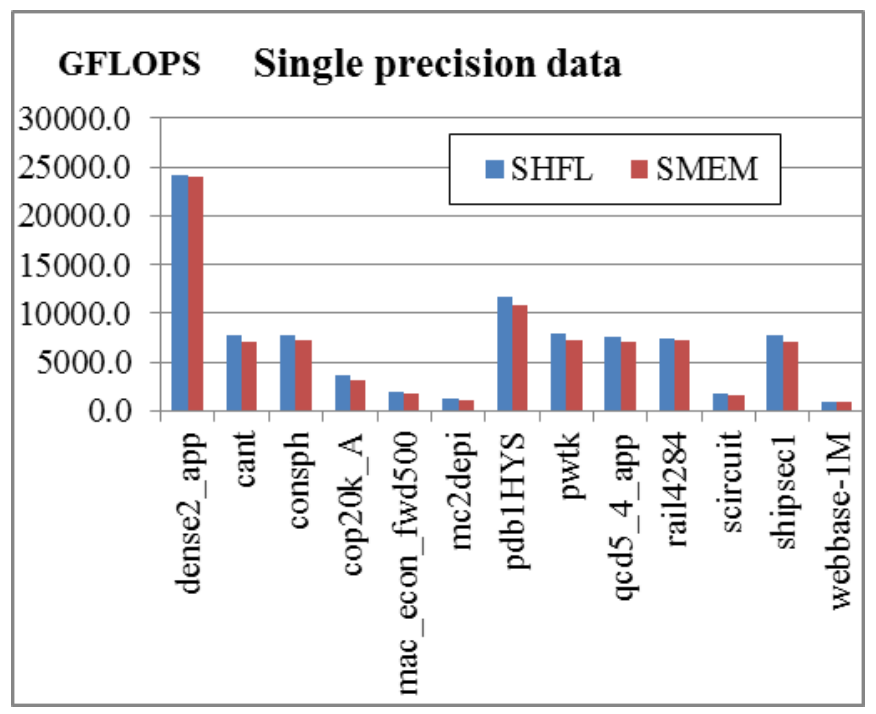

Figure 3. Throughput of Single-Precision Data

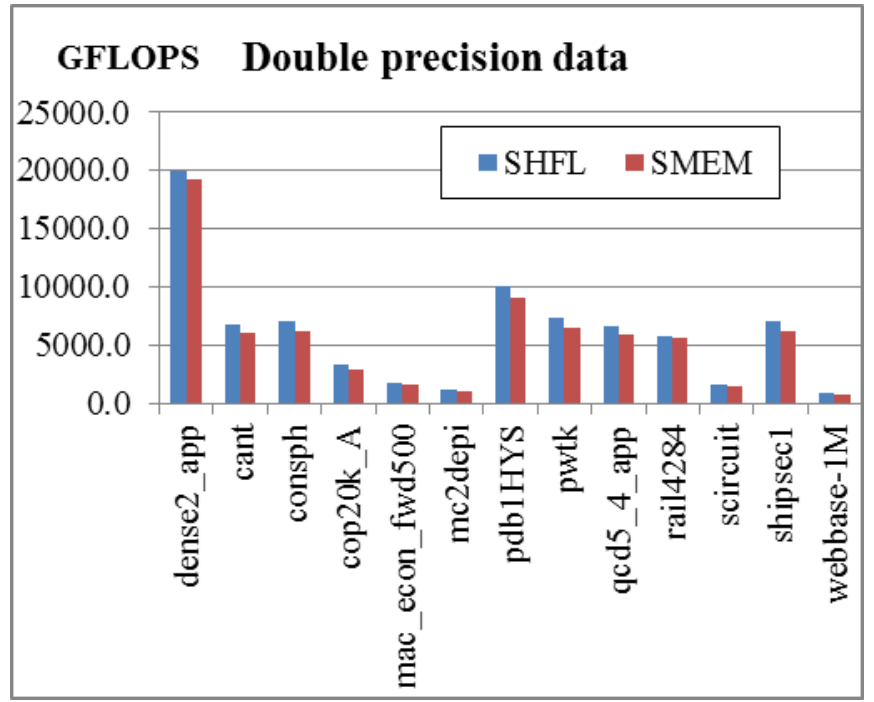

Figure 4. Throughput of double-Precision Data

Upon the experimental results, for Kepler GPU, shuffle can be more effective than shared memory for exchanging the value of register variable between threads within warp. The mainly reasons are that shuffle reduction could save to read from or write to shared memory and avoid bank conflict while multiple threads within warp accessing the shared memory simultaneously. They exchange register variable and do reduction directly, so it save a lot of time.

\section{Conclusion}

In this work, we focus on the reducing routine of SpMV of CUSP, we use shuffle reducing instead of reducing with shared memory. Experimental results show that shuffle reducing can provide much more throughput than reduction with shared memory within warp for Kepler GPU. Especially there is one more times iterating of SpMV for some applications, the improvement will be tremendous, so the latency of additional accessing shared memory should not be ignored. 


\section{Acknowledgements}

We thank for Supercomputing Center, Beihang University providing the platform to do the experiment, and we thank for Zhen Lin to discuss with me. The work is supported by the China Postdoctoral Science Foundation under Grant No. 2014M550662; the fund of the State Key Laboratory of Software Development Environment under Grant No. SKLSDE-2014KF04; the fund of the Beijing Key Lab of Intelligent Telecommunication Software and Multimedia under the project "Key technology research for the framework of Dealing with the scientific Big Data"; 2015 IBM SUR Project.

\section{References}

[1] I. S. Duff, “A Survey of Sparse Matrix Research”, Proceedings of the IEEE, vol. 65,no. 500, (1977).

[2] B. Nathan and M. Garland, "Implementing Sparse Matrix-Vector Multiplication on Throughput-Oriented Processors", Portland, USA SC, (2009) November 14-20.

[3] CUDA C PROGRAMMING GUIDE, (2012).

[4] CUDA Toolkit 5.0 CUSPARSE Library, (2012).

[5] J. Demouth, "Shuffle: Tips and Tricks", GPU Technology Conference, ; California, USA (2013) March 1821.

[6] T. Davis and Y. F. Hu, "The University of Florida Sparse Matrix". Collection.http://www.cise.ufl.edu/research/sparse/matrices/.

\section{Authors}

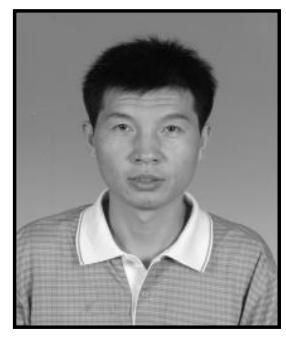

Yuan Tao, He received his ME degree in College of Information Science and Engineering from North East University in 2004, and the $\mathrm{Ph} . \mathrm{D}$. degree in computer system and architecture in Beihang University in 2015. His main research areas are parallel algorithm, parallel and distributed systems, computer system software and computer architecture.

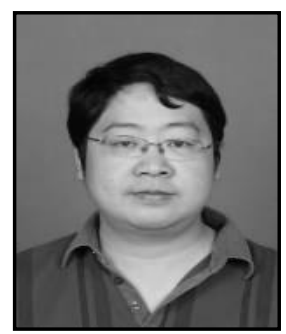

Huang Zhi-Bin, He received his Ph.D. degree in Beihang University, China in 2013. He is now a postdoctoral fellow at Beijing University of Posts and Telecommunications. He is a member of IEEE and ACM. His research interests include high performance cache architecture and multicore architecture, memory hierarchy design, high performance computation and parallel computation. 